\title{
ESTUDO ANGIOGRÁFICO DE FÍSTULA ARTERIOVENOSA UTILIZANDO GÁS CARBÔNICO COMO MEIO DE CONTRASTE*
}

\author{
José Reginaldo Simão ${ }^{1}$, Ana Terezinha Guillaumon ${ }^{2}$
}

Resumo OBJETIVO: Este estudo tem por finalidade obter um método diagnóstico angiográfico alternativo que possa ser utilizado em doentes com alto risco ao uso de meio de contraste iodado. MATERIAIS E MÉTODOS: Foram estudados 26 doentes com insuficiência renal crônica, que tiveram suas fístulas analisadas, submetidos à angiografia digital - na primeira fase, utilizando o meio de contraste iodado e, na segunda fase, gás carbônico como meio de contraste, com registro em filme angiográfico. A angiografia foi avaliada por dois médicos independentes, que analisaram a opacificação, o diagnóstico radiológico e o calibre dos vasos; a análise comparativa das medidas da artéria, da veia e da freqüência respiratória antes e após a injeção de contraste foi realizada pelo autor. RESULTADOS: Os resultados obtidos pela analise estatística utilizando coeficiente kappa apresentaram concordância entre os dois médicos, referente à opacificação, de 0,3217 , referente ao diagnóstico radiológico, de 0,5583 , e referente à analise de calibre dos vasos, de 0,4298 . A análise das medidas da artéria e da veia não apresentou diferença significativa pela medida de posição e dispersão, mostrando concordância na regressão linear, com p-valor de 0,3657 e de 0,2041; para a freqüência respiratória, as análises das medidas de posição e dispersão não apresentaram diferença significativa. CONCLUSÃO: Concluímos ser este método uma alternativa no estudo angiográfico em pacientes com antecedente alérgico ou com risco nefrotóxico.

Unitermos: Angiografia com gás carbônico; Insuficiência renal; Fístula arteriovenosa.

Abstract Arteriovenous fistula angiography using carbon dioxide as contrast medium.

OBJECTIVE: The aim of this study is to obtain an alternative angiographic diagnostic method which can be used in patients at a high risk for the use of iodine contrast medium. MATERIALS AND METHODS: Twentysix patients with chronic renal failure whose fistulas were assessed by digital angiography were first evaluated using iodine contrast medium. Subsequently, carbon dioxide was used as contrast medium, and the images were printed in angiographic film. The angiographic studies were evaluated for opacification, radiological diagnosis and vessel diameter by two independent vascular surgeons; the author performed a comparative analysis of the measurement of the artery and the vein, and respiratory frequency before and after contrast injection. RESULTS: The results obtained with the statistical analysis using kappa coefficient showed agreement between the two doctors and were 0.3217 for opacification, 0.5583 for radiological diagnosis and of 0.4298 for the analysis of vessel diameter. For the carbon dioxide technique, no significant difference was seen in the comparative analysis of the measures of the artery $(p=0.3657)$ and vein $(p=0.2041)$, in the position measurements and dispersion; the analysis of the position measurements and dispersion for respiratory frequency showed no significant difference. CONCLUSION: We concluded that this method can be used as an alternative for angiographic study in patients with history of allergy or risk of nephrotoxicity. Key words: Angiography using carbon dioxide; Renal failure; Arteriovenous fistula.

\section{INTRODUÇÃO}

A angiografia é um método diagnóstico que permitiu avanços no estudo de doen-

* Trabalho realizado na Faculdade de Ciências Médicas da Universidade Estadual de Campinas (FCM-Unicamp), com base na Dissertação de Mestrado apresentada à Pós-Graduação em Cirurgia da FCM-Unicamp.

1. Mestre em Cirurgia, Cirurgião Vascular-Angiorradiologista do Serviço de Diagnóstico por Imagem do Hospital e Maternidade Celso Pierro, Pontifícia Universidade Católica de Campinas.

2. Doutora em Cirurgia, Professora de Angiologia e Cirurgia Vascular da FCM-Unicamp.

Endereço para correspondência: Dr. José Reginaldo Simão. Rua José Domingues, 747, ap. 52, Bairro Taboão. Bragança Paulista, SP, 12900-260. E-mail: regisimao@uol.com.br

Recebido para publicação em 27/5/2003. Aceito, após revisão, em 26/11/2003. ças vasculares em todos os territórios periférico, cardíaco e neurológico - , apresentando, também, grande evolução quanto à técnica aplicada, equipamentos, cateteres e meios de contraste, diminuindo assim a morbidade e a mortalidade.

O método evoluiu desde 1896, quando Haescheck e Lindenthal introduziram uma mistura de bismuto, chumbo e bário em uma mão amputada, realizando a primeira angiografia $^{(\mathbf{1})}$. Egas Moniz, brilhante médico português, realizou a primeira punção carotídea, sendo um expoente, o que permitiu o estudo de doenças da circulação cerebral. Em 1929, Reinaldo dos Santos realiza sua técnica de aortografia abdominal com punção translombar ${ }^{(2)}$.

A partir de 1933, foram desenvolvidos novos meios de contraste, e por volta de 1950 iniciaram-se estudos que chegaram a um meio de contraste derivado da acetilação do grupo amina, de menor toxicidade, evoluindo para os meios de contraste que são utilizados até hoje ${ }^{(3)}$.

Outro marco importante, descrito por Seldinger em 1953, foi o que permitiu progredir com um cateter pela árvore arterial, também apresentando grande evolução de materiais e técnicas que tornaram possível navegar em regiões antes não alcançadas ${ }^{(4)}$. 
Toda essa evolução de conhecimentos e materiais apresentou resultado promissor na década de 1980, com a introdução da angiografia digital $^{(\mathbf{5})}$.

Porém, apesar da grande evolução, com redução da morbidade e da mortalidade, ainda se apresentavam algumas complicações relacionadas aos meios de contraste, que nos desafiaram na busca de um método adequado.

A nefrotoxicidade e a reação alérgica são os dois principais fenômenos indesejáveis quando são utilizados os contrastes à base de iodo. Apesar do uso de contrastes não-iônicos apresentar uma diminuição desses efeitos, a procura de meios de contraste que não apresentassem tais fenômenos indesejáveis tornou-se constante ${ }^{(6,7)}$.

A utilização de gás carbônico como meio de contraste em radiologia iniciouse em 1914 com Rautemberg. Em 1971, Hawkins utiliza o gás carbônico no estudo vascular periférico ${ }^{(7)}$.

Esse meio de contraste vem sendo estudado por muitos pesquisadores, o que permitiu seu emprego em vários territórios, não sendo nefrotóxico e nem produtor de efeitos alérgicos, apresentando, ainda, vantagens e desvantagens que serão discutidas no decorrer deste estudo.

\section{MATERIAIS E MÉTODOS}

Foram estudados 26 doentes, sendo dez do sexo masculino e 16 do feminino, com diagnóstico de insuficiência renal crônica, que estavam sendo submetidos à hemodiálise através de fístulas arteriovenosas.

A faixa etária foi fixada em décadas, tendo o doente mais jovem a idade de 36 anos e o mais idoso, 74 anos. Na faixa de 30 a 40 anos foram estudados dois doentes; na faixa de 41 a 50 anos, oito doentes; na faixa de 51 a 60 anos, seis doentes; na faixa de 61 a 70 anos, oito doentes; e na faixa de 71 a 80 anos, dois doentes.

Os antecedentes clínicos eram: 18 doentes com hipertensão arterial, 11 doentes com diabetes e sete doentes com aterosclerose periférica, não havendo identificação de antecedente de reação alérgica a contraste iodado.

Desse grupo de doentes, foram estudados 22 membros superiores, sendo oito direitos e 14 esquerdos, e cinco membros inferiores, sendo um direito e quatro esquerdos.

Foram estudadas oito fístulas arteriovenosas radiocefálicas, seis braquiomedianas, quatro braquiobasílicas, três braquiocefálicas, quatro alças femorais com politetrafluoretileno (PTFE) e uma alça femoral com safena.

O método utilizado consiste na cateterização seletiva do vaso sob anestesia local (técnica de Seldinger), pela artéria femoral ou artéria braquial, e progressão do cateter até próximo da fístula arteriovenosa, utilizando-se cateter multiuso $5 \mathrm{~F}$.

A angiografia foi realizada em um aparelho GE, LUA, com subtração de imagem de alta resolução.

Foram realizados estudos segmentares, iniciando-se pela artéria e progredindo distalmente até o segmento venoso. A primeira série do estudo foi realizada com contraste iodado a um volume de $10 \mathrm{ml}$ a cada injeção, utilizando-se seringa de plástico de $10 \mathrm{ml}$, de forma manual. A exposição aos raios X foi feita com 2,5 exposições/segundo. A segunda série foi realizada com gás carbônico: foi injetado um volume de 10 $\mathrm{ml}$, de forma manual, utilizando-se seringa plástica de $10 \mathrm{ml}$, sendo o gás aspirado sob pressão através de uma extensão de borracha conectada ao cilindro de gás carbônico. A exposição aos raios $\mathrm{X}$ foi feita com 7,0 exposições/segundo.

A aquisição de imagens foi realizada no sistema de angiografia digital. Outros recursos foram utilizados para aprimorar a qualidade, como, por exemplo, o contraste do vaso ser visualizado na forma negra ou branca (negativa ou positiva). Quando é

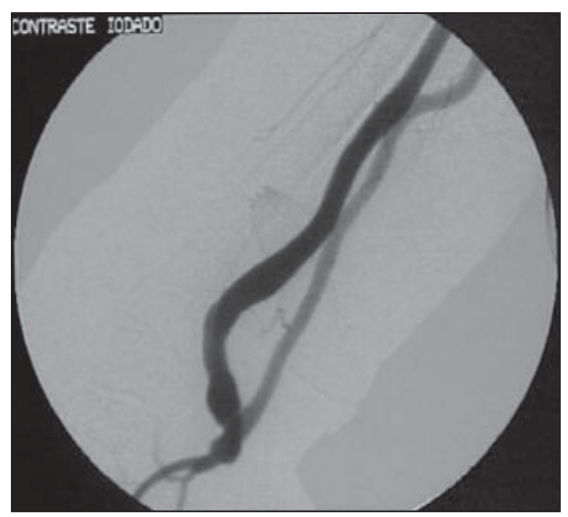

A

Figura 1. Foto da imagem angiográfica final. A: Imagem utilizando iodo como meio de contraste. B: Imagem utilizando gás carbônico como meio de contraste. usado o gás carbônico como meio de contraste, este material tende a se separar em varias bolhas. No entanto, utiliza-se a integração das imagens para corrigir essa situação - agrupar em uma única coluna de contraste.

Após o preparo das imagens, elas são impressas em filme radiológico, que apresenta as duas sequiências, com contraste iodado e com gás carbônico, utilizando-se uma processadora a laser (Figura 1).

As imagens obtidas foram impressas em filmes radiológicos, enumeradas de 1 a 26 para identificar cada doente do estudo, e entregues a dois médicos-cirurgiões vasculares e angiografistas experientes, para analisarem três aspectos: opacificação (grau de enegrecimento das imagens), diagnóstico radiológico (observação de dilatações, estenoses e/ou oclusões) e calibre dos vasos (observação da morfologia, comparando, através da opacificação dos vasos, se apresentavam o mesmo desenho). A avaliação individual da imagem de cada doente foi feita através de um formulário entregue a cada médico, em que os itens acima descritos vinham seguidos de quatro alternativas — ótimo, bom, regular e péssimo conforme a interpretação de cada um.

Após a impressão das imagens em películas, foram realizadas as medidas no segmento arterial e venoso, tanto na imagem com contraste iodado como na imagem com gás carbônico, sendo utilizada a unidade métrica de medida.

$\mathrm{Na}$ admissão dos doentes, foram tomadas medidas de freqüência respiratória de cada um, antes da entrada na sala de exame e após o término do exame, e anotadas

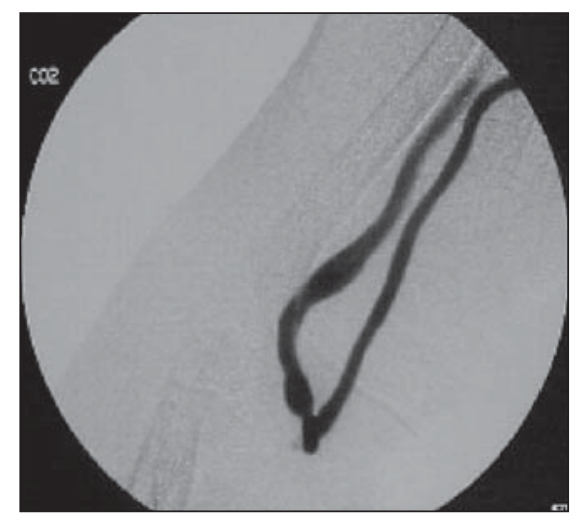
B 
em uma ficha individual, seguindo a mesma numeração de 1 a 26.

O estudo estatístico das medidas obtidas na artéria e na veia, quando foram usados os dois meios de contraste, foi realizado através da análise descritiva de medidas de posição e dispersão das variáveis. Foi utilizado, ainda, o teste t-pareado para comparação das medidas avaliadas nos dois momentos. Para verificar a concordância entre as duas medidas contínuas, foi utilizada a análise de regressão linear ${ }^{(\mathbf{8 , 9})}$.

Para o estudo estatístico das medidas da freqüência respiratória, antes e após a injeção do meio contraste, foi realizada análise descritiva através de medidas de posição e dispersão das variáveis nos dois seg$\operatorname{mentos}^{(\mathbf{8 , 9})}$.

Para verificar a concordância entre as avaliações dos dois profissionais, foi utilizado o coeficiente kappa. A interpretação da magnitude desse coeficiente é definida como: valores maiores ou iguais a 0,75 indicam excelente concordância, valores entre 0,75 e 0,40 indicam boa concordância, e valores menores ou iguais a 0,40 não indicam concordância ${ }^{(\mathbf{8 , 9})}$.

\section{RESULTADOS}

Os resultados obtidos com a análise das medidas do diâmetro dos vasos obtidos foram os seguintes:

O estudo arteriográfico com a utilização de contraste iodado mostrou que a variação de calibre na artéria foi de $1,0 \mathrm{~mm}$ a 4,0 mm, tendo média de $2,8 \mathrm{~mm}$; o estudo do segmento venoso apresentou variação de calibre na veia de $1,5 \mathrm{~mm}$ a $8,0 \mathrm{~mm}$, tendo média de 4,2 $\mathrm{mm}$ (Tabela 1).

O estudo arteriográfico com a utilização de gás carbônico como meio de contraste mostrou que a variação de calibre na artéria foi de 1,0 $\mathrm{mm}$ a 4,0 $\mathrm{mm}$, tendo média de 2,7 mm; o estudo do segmento venoso mostrou variação de calibre na veia de $1,5 \mathrm{~mm}$ a 7,5 mm, tendo média de 4,0 mm (Tabela 1).

As medidas de posição e dispersão das variáveis utilizadas pelos dois métodos não apresentaram diferença significativa, quando se mediu a artéria e a veia utilizando como meios de contraste o iodo e o gás carbônico (Tabela 1).

$\mathrm{Na}$ análise de regressão linear para medida da artéria pelos dois métodos, com contraste iodado e gás carbônico, observou-se que houve concordância entre os métodos, sendo o p-valor de 0,3657 (Tabela 2).

$\mathrm{Na}$ análise de regressão linear para medida da veia pelos dois métodos, com contraste iodado e gás carbônico, observou-se que houve concordância entre os métodos, sendo o p-valor de 0,2041 (Tabela 3).

Foram analisados os dados vitais que pudessem sofrer alterações em função do material utilizado para a execução do procedimento. Visto que o gás carbônico é eliminado pelas vias respiratórias, foi mensurada a frequiência respiratória antes e depois da injeção de contraste.

A análise de medidas de posição e dispersão da freqüência respiratória, antes e depois da realização do exame, não apre- sentou diferença significativa das medidas obtidas, tendo valor mínimo de 14 movimentos/minuto e valor máximo de 20 movimentos/minuto na fase pré-exame, e valor mínimo de 12 movimentos/minuto e valor máximo de 25 movimentos/minuto na fase pós-exame (Tabela 4).

Na segunda etapa de apreciação deste estudo, cujas imagens obtidas foram analisadas por dois cirurgiões vasculares e angiografistas, foram definidos três parâmetros, tendo como "padrão ouro" as imagens obtidas com a utilização de contraste iodado. Os parâmetros em questão foram: a) opacificação (qualidade de enegrecimento das imagens); b) diagnóstico radiológico (verificar se existem estenoses, dilatações e/ou oclusões); c) análise do calibre dos vasos (comparar as veias e artérias nos dois

Tabela 1 Medida de posição e dispersão na artéria e na veia utilizando iodo e gás carbônico como meios de contraste.

\begin{tabular}{|l|c|c|c|c|c|c|}
\hline Variável & $\mathrm{N}$ & Média & Desvio-padrão & Mínimo & Mediana & Máximo \\
\hline Art. iodo & 26 & 2,8 & 0,7 & 1,0 & 2,5 & 4,0 \\
Art. GC & 26 & 2,7 & 0,7 & 1,0 & 2,5 & 4,0 \\
Veia iodo & 26 & 4,2 & 1,8 & 1,5 & 3,8 & 8,0 \\
Veia GC & 26 & 4,0 & 1,8 & 1,5 & 3,5 & 7,5 \\
\hline
\end{tabular}

Art. iodo, artéria com iodo como meio de contraste; Art. GC, artéria com gás carbônico como meio de contraste; Veia iodo, veia com iodo como meio de contraste; Veia GC, veia com gás carbônico como meio de contraste.

Tabela 2 Resultado da análise de regressão linear para medida da artéria nos dois métodos - segundo o modelo: artéria (contraste iodado) $=a+b$ artéria (gás carbônico).

\begin{tabular}{|l|c|c|c|r|r|}
\hline \multicolumn{1}{|c|}{ Variável } & GL & Parâmetro estimado & Erro-padrão & $\mathrm{t}$ & \multicolumn{1}{c|}{$\mathrm{p}$-valor } \\
\hline Intercepto (a) & 1 & 0,19903 & 0,21585 & 0,92 & 0,3657 \\
Art. GC (b) & 1 & 0,97573 & 0,07882 & 12,38 & $<0,0001$ \\
$\mathrm{R}_{2}$ do modelo $=0,8646$ & & & & \\
$(r=0,930)$ & & & & & \\
\hline
\end{tabular}

Art. GC, artéria com gás carbônico como meio de contraste; GL, grau de liberdade.

Tabela 3 Resultado da análise de regressão linear para medida da veia nos dois métodos - segundo o modelo: veia (contraste iodado) $=a+b$ *veia (gás carbônico).

\begin{tabular}{|l|c|c|c|r|r|}
\hline \multicolumn{1}{|c|}{ Variável } & GL & Parâmetro estimado & Erro-padrão & $\mathrm{t}$ & $\mathrm{p}$-valor \\
\hline Intercepto $(\mathrm{a})$ & 1 & 0,18932 & 0,14503 & 1,31 & 0,2041 \\
Veia GC (b) & 1 & 1,00074 & 0,03301 & 30,32 & $<0,0001$ \\
$\mathrm{R}_{2}$ do modelo $=0,9746$ & & & & \\
$(\mathrm{r}=0,987)$ & & & & & \\
\hline
\end{tabular}

Veia GC, veia com gás carbônico como meio de contraste; GL, grau de liberdade.

Tabela 4 Medida de posição e dispersão da freqüência respiratória antes e após a realização do estudo.

\begin{tabular}{|l|c|c|c|c|c|c|c|}
\hline Variável & N & Média & Desvio-padrão & Mínimo & Mediana & Máximo & p-valor* \\
\hline FR pré & 26 & 16,9 & 1,8 & 14,0 & 17,0 & 20,0 & \\
FR pós & 26 & 17,6 & 2,6 & 12,0 & 17,5 & 25,0 & 0,1498 \\
\hline
\end{tabular}

* Teste t pareado. FR pré, freqüência respiratória pré-estudo; FR pós, freqüência respiratória pós-estudo. 
métodos). Esses três parâmetros foram mensurados em quatro valores: ótimo, bom, regular e péssimo.

Esse método foi utilizado por profissionais experientes e atuantes na área, independentes do estudo, para os quais foi entregue uma ficha numerada de cada doente, com os caracteres descritos acima, e os filmes radiológicos enumerados de 1 a 26 .

O primeiro parâmetro analisado pelo médico-cirurgião vascular e angiografista designado como médico 1 foi a opacificação dos vasos, utilizando gás carbônico como meio de contraste. Foi obtida, em 13 doentes, ótima qualidade $(50 \%)$; em 12 doentes, boa qualidade (46\%); e em um doente, regular qualidade (4\%). Não foi observado nenhum caso de péssima qualidade, sendo sempre feita comparação com o "padrão ouro", ou seja, o contraste iodado.

O segundo parâmetro analisado pelo médico 1 foi quanto ao diagnóstico radiológico, utilizando gás carbônico como meio de contraste. Foi obtida, em 17 doentes, ótima qualidade $(65 \%)$; em sete doentes, boa qualidade (27\%); e em dois doentes, regular qualidade (8\%). Não foi observado nenhum caso de péssima qualidade, sendo sempre feita comparação com o "padrão ouro", ou seja, o contraste iodado.

O terceiro parâmetro analisado pelo médico 1 foi quanto à análise de calibre dos vasos, utilizando como meio de contraste o gás carbônico. Foi obtida, em 14 doentes, ótima qualidade (53\%); em nove doentes, boa qualidade (35\%); e em três doentes, regular qualidade (12\%). Não foi observado nenhum caso de péssima qualidade, sendo sempre feita comparação com o "padrão ouro", ou seja, o contraste iodado.

O primeiro parâmetro analisado pelo médico-cirurgião vascular e angiografista designado como médico 2 foi a opacificação dos vasos, utilizando gás carbônico como meio de contraste. Foi obtida, em 14 doentes, ótima qualidade (54\%); em oito doentes, boa qualidade (31\%); e em quatro doentes, regular qualidade $(15 \%)$. Não foi observado nenhum caso de péssima qualidade, sendo sempre feita comparação com o "padrão ouro", ou seja, o contraste iodado.

O segundo parâmetro analisado pelo médico 2 foi quanto ao diagnóstico radio- lógico, utilizando gás carbônico como meio de contraste. Foi obtida, em 13 doentes, ótima qualidade (50\%); em 12 doentes, boa qualidade (46\%); e em um doente, regular qualidade (4\%). Não foi observado nenhum caso de péssima qualidade, sendo sempre feita comparação com o "padrão ouro", ou seja, o contraste iodado.

O terceiro parâmetro analisado pelo médico 2 foi quanto à análise de calibre dos vasos, utilizando como meio de contraste o gás carbônico. Foi obtida, em 13 doentes, ótima qualidade $(50 \%)$; em 11 doentes, boa qualidade (42\%); e em dois doentes, regular qualidade $(8 \%)$. Não foi observado nenhum caso de péssima qualidade, sendo sempre feita comparação com o "padrão ouro", ou seja, o contraste iodado.

$\mathrm{Na}$ análise de concordância quanto à opacificação pelos dois médicos angiografistas, utilizando o coeficiente kappa ponderado, obteve-se coeficiente de 0,3217, não indicando concordância entre os dois.

$\mathrm{Na}$ análise de concordância quanto ao diagnóstico radiológico pelos dois médicos angiografistas, utilizando o coeficiente kappa ponderado, obteve-se coeficiente de 0,5583, indicando boa concordância entre os dois.

Na análise de concordância quanto à analise de calibre dos vasos feita pelos dois médicos angiografistas, utilizando o coeficiente kappa ponderado, obteve-se coeficiente de 0,4298, indicando boa concordância entre os dois.

\section{DISCUSSÃO}

A angiografia teve grande evolução em sua técnica radiológica, técnicas de cateterismo e meios de contraste. Apesar de tal evolução, o método diagnóstico ainda apresenta restrições ao uso de contraste iodado em doentes com insuficiência renal incipiente. A nefrotoxicidade com o uso de contraste iodado varia de $0 \%$ a $27 \%$, podendo essas taxas ser superiores, dependendo de lesão renal prévia(10-12).

Os antecedentes de atopia ao iodo também apresentam restrições quanto ao uso dessa substância, com taxas que variam de $4 \%$ a $7 \%$, aumentando para $38 \%$ quando o doente relata história de reação alérgica anterior $^{(\mathbf{3 , 1 2})}$.
A utilização do gás carbônico como meio de contraste tem como principais vantagens não apresentar reação alérgica e nem ser lesiva ao parênquima renal, fato discutido na grande maioria dos estudos realizados em doentes de alto risco à utilização de contraste iodado ${ }^{(\mathbf{4 , 6}, 13-19)}$.

A utilização do gás carbônico apresenta como limitação o efeito neurotóxico, porém os estudos realizados ainda não são conclusivos, o que limita seu uso ao estudo dos troncos supra-aórticos e vasos cerebrais. Essas reações adversas não foram objetivos deste estudo, visto que, quanto à nefrotoxicidade, os doentes que foram estudados já apresentavam doença renal, sendo submetidos à hemodiálise. E, quanto à reação alérgica, ela não foi constatada em nenhum doente. Neste estudo, quando a angiografia foi realizada nos membros superiores, o cateter era avançado além da artéria vertebral, de modo a se evitar que o gás carbônico pudesse progredir para o

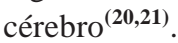

Para demonstrar a eficácia desse método, foram utilizados parâmetros que pudessem expressar os resultados obtidos, sendo que o resultado final utilizando gás carbônico como meio de contraste, comparado com o meio de contraste iodado, apresentou suficiente qualidade de imagem, demonstrando ser um método alternativo no estudo angiográfico. Apresenta vantagens no seu uso, por ser não nefrotóxico, não alérgico, de baixa viscosidade, permitindo que se utilizem cateteres de diâmetro bem inferior aos utilizados na prática. Também é utilizado nos procedimentos intervencionistas, apresentando, ainda, mínimo desconforto e baixo custo. Porém, para se obter imagens de qualidade, há a necessidade de equipamento adequado - angiografia digital com subtração de imagem e programas que permitam trabalhar as imagens adquiridas ${ }^{(\mathbf{8 , 2 2 , 2 3})}$.

Após a aquisição das imagens, utilizando meio de contraste iodado e gás carbônico, foram realizadas medidas dos diâmetros vasculares, tendo como unidade de medida o metro. Para a análise estatística, foram realizadas medidas de posição e dispersão das variáveis pelos dois métodos, não apresentando variações significativas nas medidas das artérias e das veias quando comparados os dois métodos. Foi tam- 
bém utilizada a regressão linear para comparar as medidas encontradas na artéria e na veia, na utilização dos dois meios de contraste, apresentando concordância das medidas obtidas, com maior concordância quando foi analisado o segmento venoso. Com esses dois métodos estatísticos observa-se, neste estudo, que os resultados encontrados, ao se realizarem as medidas da artéria e da veia, mostraram que o gás carbônico pode ser utilizado como meio de contraste alternativo.

Confrontando com dados da literatura, quando foi analisado o grau de estenose dos vasos com utilização de gás carbônico e contraste iodado, neste estudo foram obtidos resultados concordantes quanto à quantificação das estenoses. Porém, em outros dois estudos — o primeiro realizado em um modelo aorto-ilíaco, utilizando os dois métodos e realizando medidas dos segmentos, e no segundo realizando implante de filtro de veia cava - , obtiveramse também variações de medidas significativas, mas sem apresentarem importância clínica no procedimento realizado ${ }^{(\mathbf{2 4 , 2 5})}$.

Quando se realiza um procedimento intervencionista, torna-se necessário obter a medida dos vasos para implante das próteses desejadas, e, na maioria dos estudos, esses procedimentos foram realizados com sucesso utilizando o gás carbônico como meio de contraste ${ }^{(24,26-29)}$.

A segunda etapa do estudo consistiu da análise das imagens obtidas por dois profissionais da área. Eles a fizeram sob três aspectos: opacificação, diagnóstico radiológico e calibre dos vasos. A análise estatística utilizada para validar essa etapa do estudo foi verificar se existia concordância dos resultados entre os dois profissionais, utilizando o coeficiente kappa, sendo este coeficiente de excelente concordância quando se apresentavam resultados maiores ou iguais a 0,75 , de boa concordância com valores entre 0,75 e 0,4 , e de nula concordância com valores inferiores a 0,4.

O primeiro tópico da avaliação do estudo foi a opacificação dos vasos, isto é, o grau de enegrecimento das imagens. Obteve-se, nessa etapa, o coeficiente ponderado kappa de 0,3217 , indicando que não houve concordância entre o médico 1 e o médico 2. Porém, quando foram comparados os resultados obtidos, o médico 1 en- controu 25 doentes entre ótimo e bom $(96 \%)$ e um doente regular (4\%), e o médico 2 classificou 22 doentes entre ótimo e bom $(85 \%)$ e quatro doentes em regular (15\%). Com base nesses dados, e apesar de não apresentar concordância pelo índice kappa, já que a análise foi subjetiva e dependente da análise visual da escala de cinzas observada por cada profissional, esses valores mostraram que houve um resultado significativo em porcentuais entre ótimo e bom quando comparados com o "padrão ouro" do estudo.

Dados encontrados na literatura relativos à comparação de arteriografia utilizando gás carbônico como meio de contraste, tendo como "padrão ouro" o contraste iodado, e que quantificaram a qualidade de imagem, apresentam variações de qualidade de imagens, dependendo do segmento estudado. Quando as angiografias são realizadas para isquemia de membros inferiores, as imagens obtidas abaixo dos joelhos apresentam qualidade inferior quando comparadas ao contraste iodado. Já em outros territórios, tanto no segmento arterial quanto no venoso, são obtidas imagens com boa qualidade técnica, podendo ser levemente inferior, mas permitindo que tais imagens sejam conclusivas.

Esses dados são concordantes com este estudo, apesar de não haver concordância entre os dois observadores. Ao apresentar um alto porcentual entre bom e ótimo, este estudo permite confirmar que houve boa qualidade de imagem. A melhoria da imagem pode ser obtida ao se utilizarem programas do equipamento de arteriografia, que tornam possível somar as imagens adquiridas, ou ao se progredir o cateter mais distalmente possível, fato que foi observado no início deste estudo. Progredindo o cateter, a tendência das bolhas de gás carbônico separarem-se diminuía, obtendose, dessa forma, uma melhor imagem.

Outro fato observado neste estudo e na literatura é que não havia melhoria da imagem com volumes de gás carbônico maiores que $10 \mathrm{ml}$. Quando utilizada a bomba ejetora, o resultado final da imagem era melhor. Neste estudo, porém, o contraste foi injetado de forma manual ${ }^{(25,30-32)}$.

O segundo tópico da avaliação do estudo foi o diagnóstico radiológico, quando os profissionais avaliaram dilatações, estenoses e/ou oclusões. Obteve-se, nessa etapa, o coeficiente ponderado kappa de 0,5583, indicando que houve concordância entre os dois profissionais. Quando foram comparados os resultados obtidos pelo médico 1, apresentavam-se 24 doentes entre ótimo e bom (92\%) e dois doentes em regular ( $8 \%$ ), e pelo médico 2 , apresentavam-se 25 doentes entre ótimo e bom (96\%) e um doente em regular (4\%). Comparando-se esses valores, demonstra-se que houve um resultado significativo de porcentuais entre ótimo e bom. Nessa etapa do estudo, a concordância já se torna mais importante, visto que o que está sendo analisado é o diagnóstico — se há dilatação, estenose ou oclusão de um determinado segmento.

Os resultados encontrados no presente estudo mostram-se semelhantes aos da literatura. O gás carbônico é um meio de contraste que permite realizar diagnósticos radiológicos com precisão, resultado que foi encontrado nos vários estudos realizados no diagnóstico de fístulas arteriovenosas, tumores, hemorragias, isquemias dos membros, aneurismas e doenças renovasculares. Também foram encontrados resultados superiores ao contraste iodado em alguns estudos com tumores, "shunt" arteriovenoso e hemorragia, pelo fato do gás carbônico apresentar baixa viscosidade. Quando um estudo apresenta baixa qualidade de opacificação, é de se esperar que apresente dificuldade de visualização fato que ocorreu nos estudos para isquemia dos membros inferiores — , apresentando, dessa forma, também baixa qualidade de diagnóstico ${ }^{(33-36)}$.

O terceiro tópico da avaliação deste estudo foi a análise de calibre dos vasos, quando os profissionais compararam a morfologia, ou seja, a visualização de todos os vasos. Obteve-se, nessa etapa, o coeficiente ponderado kappa de 0,4 , indicando que houve concordância entre os dois profissionais. Quando foram comparados os resultados obtidos pelo médico 1 , apresentavam-se 23 doentes entre ótimo e bom $(88 \%)$ e três doentes em regular (12\%), e pelo médico 2, apresentavam-se 24 doentes entre ótimo e bom $(92 \%)$ e dois doentes em regular (8\%). Essa análise também foi visual. Comparando-se esses valores, demonstra-se que houve também um 
resultado significativo em porcentuais entre ótimo e bom. Essa fase do estudo igualmente apresentou concordância quando comparados seus dados com os encontrados na literatura.

As imagens obtidas das artérias proximais eram equivalentes nas duas técnicas. Porém, quando analisadas as imagens das artérias abaixo do joelho, elas apresentavam qualidade inferior quando comparadas à angiografia realizada com contraste iodado - fato observado neste estudo que pode ser melhorado ao se progredir o cateter distalmente à artéria. Outros estudos conseguiram visualização até a terceira divisão dos vasos. Em alguns deles, o gás carbônico mostrou resultados superiores ao contraste iodado - o que ocorreu nos estudos de tumores, "shunt" hepático e fístulas arteriovenosas —, apresentando visualização de vasos que não mostravam opacificação com contraste iodado, fato também observado neste estudo. Em virtude da menor viscosidade do gás carbônico, ele apresenta maior tendência de se dirigir para os vasos onde há maior velocidade de fluxo ${ }^{(37-41)}$.

Outro tópico analisado no estudo foi a freqüência respiratória, expressa em movimentos/minuto, que foi verificada antes de o doente entrar na sala de exame e depois do término do exame. Esta análise foi realizada com o objetivo de comprovar que a infusão do gás carbônico não causava alterações significativas da fisiologia respiratória. Esses dados foram colocados em planilha e, posteriormente, submetidos à análise estatística, com medidas de posição e dispersão nos dois momentos, com teste tpareado e $\mathrm{p}=0,1498$, evidenciando não haver variação significativa entre os dois. Portanto, não houve variação da freqüência respiratória após a injeção de gás carbônico para a execução do estudo. Resultados obtidos na literatura confirmam os aqui encontrados. Yusuf et al., em seu estudo analisando $\mathrm{pH}, \mathrm{PaCO}_{2}$ e $\mathrm{PO}_{2}$ em arteriografia com gás carbônico, não encontraram variação significativa desses gases, concluindo que o gás carbônico era eliminado sem causar qualquer complicação ${ }^{(\mathbf{1 2})}$.

Esse gás é de ligação fraca, sendo transportado na corrente sanguínea, na sua maior parte, na forma de bicarbonato, e uma pequena parte, transportado na forma de gás carbônico livre. Sua difusão é cerca de 20 vezes maior que a do oxigênio, o que facilita sua liberação para as vias res-

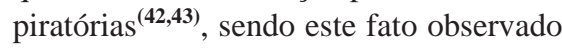
quando, logo após sua injeção, havia uma tendência da coluna de contraste de se separar em vários aglomerados e desaparecer à medida que o fluxo progredia distalmente ao vaso estudado.

Neste estudo, as sequiências realizadas com gás carbônico foram executadas de forma manual, injetando-se $10 \mathrm{ml}$, com seringas de $10 \mathrm{ml}$, em intervalos de tempo de aproximadamente 60 segundos entre cada injeção. A velocidade de difusão do gás carbônico em repouso é cerca de 400 $\mathrm{ml} / \mathrm{min}$, chegando a $1.300 \mathrm{ml} / \mathrm{min}$ durante o exercício. Com base nesses dados, comparando a diferença da frequiência respiratória nas duas situações e o volume utilizado neste estudo, que é de aproximadamente $10 \mathrm{ml} / \mathrm{min}$, observamos que esse valor é bem inferior à diferença do valor encontrado nas duas situações mencionadas $\operatorname{acima}^{(\mathbf{4 2}, 43)}$.

\section{CONCLUSÃO}

Foram propostos aqui cinco parâmetros para avaliação que possibilitariam apresentar uma conclusão da viabilidade deste método: a opacificação dos vasos, o diagnóstico radiológico, a análise de calibre, a análise comparativa das medidas da artéria e da veia com os dois métodos e a freqüência respiratória antes e depois do exame. Todos esses aspectos apresentaram resultados significativos utilizando gás carbônico, comparado com o "padrão ouro", que é meio de contraste iodado.

Diante dos argumentos apresentados, permite-se concluir ser o uso do gás carbônico um método alternativo no estudo angiográfico digital em doentes com fístulas arteriovenosas para realização de hemodiálise.

\section{REFERÊNCIAS}

1. Eisenberg RL. Roentgen and the discovery of Xrays. In: Eisenberg RL, ed. Radiology: an illustrated history. St. Louis, MO: Mosby Year Book, 1992: 22-42.

2. Fecuri Jr R. Arteriografia convencional. In: Maffei FHA, Lastória S, Yoshida WB, Rollo HA. Doenças vasculares periféricas. $3{ }^{a}$ ed. Rio de Janeiro, RJ: Medsi, 2002:368-81.

3. Yoshida WB. Contrastes utilizados em angiogra- fias. In: Maffei FHA, Lastória S, Yoshida WB, Rollo HA. Doenças vasculares periféricas. $3^{\underline{a}} \mathrm{ed}$. Rio de Janeiro, RJ: Medsi, 2002:360-5.

4. Araujo AP, Lopez GE, Gomes CPA. Fios-guias, introdutores e cateteres para angiografia e procedimentos endovasculares. In: Maffei FHA, Lastória S, Yoshida WB, Rollo HA. Doenças vasculares periféricas. $3 \underline{\underline{a}}$ ed. Rio de Janeiro, RJ: Medsi, 2002: $347-65$.

5. Moura R. Angiografia por subtração digital. In: Maffei FHA, Lastória S, Yoshida WB, Rollo HA. Doenças vasculares periféricas. $3^{\mathbf{a}} \mathrm{ed}$. Rio de Janeiro, RJ: Medsi, 2002:383-98.

6. Back MR, Caridi JG, Hawkins IF Jr, Seeger JM. Angiography with carbon dioxide $\left(\mathrm{CO}_{2}\right)$. Surg Clin North Am 1998;8:575-91.

7. Kerns SR, Hawkins IF Jr, Sabatelli FW. Current status of carbon dioxide angiography. Radiol Clin North Am 1995;33:15-29.

8. Agresti A, Finlay B. Statistical methods for the social sciences. San Francisco: Dellen Publishing Co., 1986.

9. Fleiss JL. Statistical methods for rates and proportions. 2nd ed. New York, NY: Wiley-Interscience, 1981.

10. Laranja SMR, Schor N. Nefrotoxicidade por contrastes radiológicos. In: Schor N, Boim MA, Santos OFP. Insuficiência renal aguda - fisiopatologia clínica e tratamento. São Paulo, SP: Sarvier, 1997: 129-32.

11. Murphy SW, Barrett BJ, Parfrey PS. Contrast nephropathy. J Am Soc Nephrol 2000;11:177-82.

12. Yusuf SW, Whitaker SC, Hinwood D, et al. Carbon dioxide: an alternative to iodinated contrast media. Eur J Vasc Endovasc Surg 1995;10:156-61.

13. Barbey MM, Farber A, Marienhoff N, Gmelin E. Digital subtraction angiography with carbon dioxide: basic principles, technique and clinical application. Vasa 1999;28:243-9.

14. Beese RC, Bees NR, Belli AM. Renal angiography using carbon dioxide. Br J Radiol 2000;73:3-6.

15. Christensson A. Renovascular disease and renal insufficiency: diagnosis and treatment. Scand J Urol Nephrol 1999;33:400-5.

16. Frankhouse JH, Ryan MG, Papanicolaou G, Yellin $\mathrm{AE}$, Weaver FA. Carbon dioxide/digital subtraction arteriography-assisted transluminal angioplasty. Ann Vasc Surg 1995;9:448-52.

17. Gahlen J, Hansmann J, Schumacher H, Seelos R, Richter GM, Allenberg JR. Carbon dioxide angiography for endovascular grafting in high-risk patients with infrarenal abdominal aortic aneurysms. J Vasc Surg 2001;33:646-9.

18. Kuo PC, Petensen J, Semba C, Alfrey EJ, Dafoe DC. $\mathrm{CO}_{2}$ angiography - a technique for vascular imaging in renal allograft dysfunction. Transplantation 1996;61:652-4.

19. Spinosa DJ, Matsumoto AH, Angle JF, Hagspiel KD, McGraw JK, Ayers C. Renal insufficiency: usefulness of gadodiamide-enhanced renal angiog raphy to supplement $\mathrm{CO}_{2}$-enhanced renal angiography for diagnosis and percutaneous treatment. Radiology 1999;210:663-72.

20. Dimakakos PB, Stefanopoulos T, Doufas AG, et al. The cerebral effects of carbon dioxide during digital subtraction angiography in the aortic arch and its branches in rabbits. AJNR 1998;19:261-6.

21. Linstedt U, Link J, Grabener M, Kloess W. Effects of selective angiography of the carotid artery with carbon dioxide on electroencephalogram somatosensory evoked potentials and histopathologic 
findings. A pilot study in pigs. Invest Radiol 2000; 32:507-10.

22. Hawkins IF, Caridi JG. Carbon dioxide $\left(\mathrm{CO}_{2}\right)$ digital subtraction angiography: 26-year experience at the University of Florida. Eur Radiol 1998;8:391402.

23. Kerns SR, Hawkins IF Jr. Carbon dioxide digital subtraction angiography: expanding applications and technical evolution. AJR 1995;164:735-41.

24. Sing RF, Stackhouse DJ, Jacobs DG, Heniford BT Safety and accuracy of bedside carbon dioxide cavography for insertion of inferior vena cava filters in the intensive care unit. J Am Coll Surg 2001; 192:168-71.

25. Song K, Cho D, Shinn K, Charlton E, Cho, K. Gas dynamics in $\mathrm{CO}_{2}$ angiography: in vitro evaluation in a circulatory system model. Invest Radiol 1999;34:151-5.

26. Black CM, Lang EV, Kusnick CA, et al. 1996 AUR Memorial Award. Densitometric analysis of eccentric vascular stenoses: comparison of $\mathrm{CO}_{2}$ and iodinated contrast media. Acad Radiol 1996;3:98593.

27. Caridi JG, Stavropoulos SW, Hawkins IF Jr. $\mathrm{CO}_{2}$ digital subtraction angiography for renal artery angioplasty in high-risk patients. AJR 1999;173: 1551-6.

28. Ehrman KO, Taber TE, Gaylord GM, Brown PB, Hage JP. Comparison of diagnostic accuracy with carbon dioxide versus iodinated contrast material in the imaging of hemodialysis access fistulas. J Vasc Interv Radiol 1994;5:771-5.
29. Moresco KP, Patel NH, Namyslowski Y, Shah H, Johnson MS, Trerotola SO. Carbon dioxide angiography of the transplanted kidney: technical considerations and imaging findings. AJR 1998;171: 1271-6.

30. Bees NR, Beese RC, Belli AM, Buckenham TM. Carbon dioxide angiography of the lower limbs: initial experience with an automated carbon dioxide injector. Clin Radiol 1999;54:833-8.

31. Oliva VL, Denbow N, Therasse E, et al. Digital subtraction angiography of the abdominal aorta and lower extremities: carbon dioxide versus iodinated contrast material. J Vasc Interv Radiol 1999;10: 723-31.

32. Schmitz-Rode T, Alzen G, Gunther RW. Digital subtraction angiography with carbon dioxide using a new gas dosage system. Rofo 1997;167:71-8.

33. Garbagnati F, Spreafico C, Marchiano A, et al. Carbon dioxide digital subtraction angiography in oncological-interventional radiology. Tumori 1995;81:52-5.

34. Hawkins IF Jr, Wilcox CS, Kerns SR, Sabatelli FW. $\mathrm{CO}_{2}$ digital angiography: a safer contrast agent for renal vascular imaging? Am J Kidney Dis 1994; 24:685-94.

35. Schreier DZ, Weaver FA, Frankhouse J, et al. A prospective study of carbon dioxide-digital subtraction vs standard contrast arteriography in the evaluation of the renal arteries. Arch Surg 1996; 131:503-7.

36. Textor HJ, Wilhelm K, Strunk H, Schuller H, Schild $\mathrm{HH}$. The diagnosis of intra-abdominal hemorrhages with $\mathrm{CO}_{2}$ as the contrast medium. Rofo 1997;166: 51-3.

37. Gorriz E, Carreira JM, Reyes R, et al. $\mathrm{CO}_{2}$ as a contrast medium in endoluminal treatment of high flow vascular malformations. Eur J Radiol 1999 31:182-7.

38. Miyazono N, Inoue H, Kanetsuki I, Nakajo M Retrograde visualization of the portal venous system using $\mathrm{CO}_{2}$ intraarterial digital subtraction angiography. Abdom Imaging 1994;19:330-3.

39. Nicolini A, Lovaria A, Meregaglia D, Palatresi S Carbon dioxide angiography. A new injection system. Radiol Med 2000;99:51-5.

40. Rolland Y, Duvauferrier R, Lucas A, et al. Lower limb angiography: a prospective study comparing carbon dioxide with iodinated contrast material in 30 patients. AJR 1998;171:333-7.

41. Teshima Y, Iwasaki N. Efficacy of $\mathrm{CO}_{2}$-DSA in embolization. Cancer Chemother Pharmacol 1994 33:S109-10.

42. Guyton AC, Hall JE. Princípios físicos das trocas gasosas; difusão do oxigênio e do dióxido de carbono através da membrana alveolar. In: Guyton AC, Hall JE, eds. Tratado de fisiologia médica. 9 ed. Rio de Janeiro, RJ: Guanabara Koogan, 1996 453-63.

43. Guyton AC, Hall JE. Transporte de oxigênio e de dióxido de carbono no sangue e em outros líquidos corporais. In: Guyton AC, Hall JE, eds. Tratado de fisiologia médica. 9a ed. Rio de Janeiro, RJ: Guanabara Koogan, 1996:455-74. 